\title{
URINARY TRACT INFECTION
}

Urinary tract infection (UTI) refers to a spectrum of clinical presentations ranging from asymptomatic urinary infection to acute pyelonephritis with septicemia. UTI is a common infection diagnosed at the time of admission or acquired during hospitalization. According to the Healthcare Cost and Utilization Project (HCUP), the Diagnosis Related Group for UTI with complications or co-morbidities accounted for almost 302,000 hospital discharges in 2002. The mean length-of-stay was 4.9 days with mean charges of $\$ 13,000$ per patient. In-hospital mortality was $2.2 \%$ for this group. Hospitalists diagnose, treat and identify complications of UTI. Hospitalists can lead hospital-wide patient safety initiatives to reduce the incidence of hospital-acquired infection and emerging antibiotic resistance.

\section{KNOWLEDGE}

Hospitalists should be able to:

- Define UTI and describe the pathophysiology that leads to complicated UTI.

- Describe common symptoms and signs of UTI.

- Explain the clinical spectrum of UTI including patient populations that may present with atypical symptoms.

- Name common community-acquired and hospital-acquired urinary pathogens.

- Explain how local and national resistance patterns impact the selection of initial antibiotics.

- Distinguish UTI from sterile pyuria and from colonization.

- Explain the indications and limitations of specific tests used to diagnose UTI, its underlying causes and complicating conditions.

- Define risk factors for UTI.

- Name specific patient populations at increased risk for development of hospital acquired or other complicated UTIs.

- Distinguish the specific clinical management, including antibiotic selection for different patient populations, including patients with community-acquired UTI, hospital-acquired UTI, chronic indwelling catheters, pregnancy, immunosuppression and incidentally recognized UTI.

- Explain the indications for hospitalization.

- Explain goals for hospital discharge, including specific measures of clinical stability for safe care transition.

\section{SKILLS}

Hospitalists should be able to:

- Elicit a targeted history to identify risk factors and symptoms for UTI and its known complications.

- Perform a focused physical examination looking for signs of complicated UTI, prostatitis and other co-morbid conditions.

- Order and interpret urinalysis and urine culture.

- Order and interpret the results of imaging studies when indicated.

- Formulate an initial care plan based on patient risk factors, acute medical illness, co-morbid disease, and local and national antibiotic resistance patterns.

- Adjust antibiotic therapy based on subsequent culture results and determine appropriate duration of treatment.

- Recognize and address complications of UTI and/or inadequate response to therapy.

- Evaluate and treat patients for UTI in the perioperative setting when indicated.

\section{ATTITUDES}

Hospitalists should be able to:

- Communicate with patients and families to explain the goals of care plan, discharge instructions and management after release from hospital.

- Communicate with patients and families to explain tests and procedures, and the use and potential side effects of pharmacologic agents.

- Recognize indications for specialty consultation, which may include urology or infectious disease services.

- Promote and employ prevention measures, which may include early removal of urinary catheters and other interventions to prevent recurrent UTI. 
- Apply judicious antibiotic selection to help reduce antibiotic resistance.

- Employ a multidisciplinary approach to the care of patients with complicated UTI that begins on admission and continues through all care transitions.

- Appreciate and treat patient's pain.

- Document treatment plan, and provide clear discharge instructions for the receiving primary care physician, including duration of antibiotic treatment and need for follow-up testing.

- Provide and coordinate resources to patients to ensure safe transition from the hospital to arranged follow-up care.

- Coordinate discharge plans when patients will require ongoing skilled nursing care.

- Utilize evidence based recommendations for the diagnosis and treatment of UTI.

\section{SYSTEM ORGANIZATION AND IMPROVEMENT}

To improve efficiency and quality within their organizations, Hospitalists should:

- Implement systems to ensure hospital-wide adherence to national standards, and document those measures as specified by recognized organizations.

- Collaborate with local infection control practitioners to reduce the spread of resistant organisms within the institution.

- Lead, coordinate or participate in multidisciplinary initiatives to minimize use and duration of urinary catheters and reduce incidence of hospital-acquired UTI. 